\title{
Reappraisal of the use of procarbazine in the treatment of lymphomas and brain tumors
}

\author{
Jean-Pierre Armand' \\ Vincent Ribrag' \\ Jean-Luc Harrousseau' \\ Lauren Abrey ${ }^{2}$ \\ 'Institut Gustave-Roussy, Desmolins, \\ Villejuif Cedex, France; ${ }^{2}$ Memorial \\ Sloan-Kettering Cancer Center, New \\ York, New York, USA
}

\begin{abstract}
Procarbazine $\mathrm{HCl}$ is a 'nonclassical' oral alkylating anticancer agent that was first synthesized in the late 1950s. It has been used in the treatment of many cancers, but its main use is in the treatment of Hodgkin's lymphoma and brain tumors and, to a lesser extent, Non-Hodgkin's lymphoma and primary central nervous system lymphoma. Procarbazine is a prodrug that undergoes metabolic transformation into active intermediates that are thought to inhibit DNA, RNA, and protein synthesis. Early use of procarbazine in combination with mechlorethamine, vincristine, and prednisone (MOPP) was effective in the treatment of advanced Hodgkin's lymphoma, but late toxic effects such as secondary cancer and infertility led to its replacement by other regimens. However, its recent reintroduction in the dose-intensified BEACOPP (bleomycin, etoposide, doxorubicin, cyclophosphamide, vincristine, procarbazine, and prednisone) regimen has yielded very promising findings. Procarbazine alone, or more commonly combined in the PCV (procarbazine, lomustine [CCNU], and vincristine) regimen, is also effective in treating gliomas comprising astrocytomas, glioblastomas, and oligodendrogliomas. The most common side effects of procarbazine are gastrointestinal disturbances, myelosuppression, and central nervous system effects. In conclusion, the use of procarbazine in combination with other drugs means that it remains a major anticancer drug in the management of Hodgkin's lymphoma and gliomas.
\end{abstract}

Keywords: procarbazine, Hodgkin's lymphoma, non-Hodgkin's lymphoma, lymphoma, brain tumor, glioma

\section{Introduction}

Procarbazine $\mathrm{HCl}$ is a 'nonclassical' oral alkylating agent that belongs to the same family as dacarbazine and hexamethylamine (Zeller et al 1963). It contains an N-methyl group that is essential for its activity, but not the chloroethyl group that is present in nitrogen mustard-type alkylating agents (Newell et al 1987). It was first synthesized in the late 1950s during a search for a new monoamine oxidase inhibitor, but was soon developed as an anticancer agent (Kenis et al 1966; Martin and Schubert 1966; Livingston and Carter 1970; Friedman 2001) and has since been used in the treatment of many cancers including Hodgkin's lymphomas, non-Hodgkin's lymphoma, brain tumors, multiple myeloma, primary central nervous system lymphoma, malignant melanoma, and lung cancer.

Procarbazine was first granted approval in 1965 and was subsequently marketed in around 84 countries. It has been widely deployed in the treatment of a number of cancers and a considerable body of clinical experience with this drug has accumulated. The introduction of safer combination therapies led to the gradual supplantation of procarbazine. During recent years, there has been a re-emergence of interest in procarbazine combinations with other chemotherapeutic agents, specifically for the treatment of Hodgkin's lymphoma and gliomas and, to a lesser extent, non-Hodgkin's lymphoma and primary central nervous system lymphoma. This is partly prompted by 
the unique mechanism of action of procarbazine; this agent has multiple sites of action and is not cross-resistant with other alkylating agents, cytostatics or radiotherapy (Matche et al 1963; Brunner and Young 1965).

Reintroduction of procarbazine in the dose-intensified BEACOPP (bleomycin, etoposide, doxorubicin, cyclophosphamide, vincristine, procarbazine, and prednisone) regimen has yielded impressive therapeutic results in the treatment of Hodgkin's lymphoma and, in combination with lomustine (CCNU) and vincristine in the PCV (procarbazine, CCNU, and vincristine) regimen, it shows considerable promise in the treatment of gliomas.

\section{Preclinical studies Pharmacology}

Procarbazine is a prodrug that undergoes a complex metabolic transformation into active intermediates. Although these intermediates have been characterized, their precise mechanism of action remains unclear. They are thought to inhibit DNA, RNA, and protein synthesis (Berneis et al 1963; Rutinshauser and Bollag 1963). Studies suggest that procarbazine may inhibit transmethylation of the methyl groups of methionine into t-RNA; the subsequent lack of functional t-RNA could cause cessation of protein synthesis, and consequently DNA and RNA synthesis. DNA may also be damaged directly by hydrogen peroxide, formed during auto-oxidation of procarbazine, which may attack the protein sulphydryl groups in residual protein that is tightly bound to DNA. These effects are specific for the $\mathrm{S}$ and $\mathrm{G} 2$ phases of the cell cycle. Drug resistance mechanisms to procarbazine also indicate that the methylating pathway is important in its mechanism of action and may help to develop new therapeutic strategies. Potential resistance mechanisms involve $\mathrm{O}^{6}$ alkylguanine DNA alkyltransferase (AGT)-mediated repair of $\mathrm{O}^{6}$-methylguanine activity and hMSH2 mismatch-repair deficiency (Pegg 1990; Friedman et al 1997).

In vivo, significant antitumor activity has been demonstrated against Ehrlich carcinoma and Crocker sarcoma S180 in mice, and Walker carcinoma 256, Guerin uterine carcinoma T8, and intracerebral leukemia L1210 in rats (Bollag and Grunberg 1963).

\section{Toxicology}

Mutagenic effects of procarbazine have been demonstrated in a variety of bacterial and mammalian test systems, and it has been shown to inhibit fetal development in rats (Malek et al 2003). Unscheduled DNA synthesis in the testes of rabbits has also been reported (Zbinden 1980). As might be expected with an agent that inhibits DNA, RNA, and /or protein synthesis, reduced fertility has been seen in male mice given procarbazine (Chryssanthou et al 1983).

Carcinogenic effects of procarbazine have been reported in mice, rats, and monkeys.

\section{Pharmacokinetics and drug disposition}

Procarbazine is quickly and completely absorbed from the gastrointestinal tract following oral administration, with peak plasma levels achieved after 0.5-1 hour (Supko et al 2002; Preiss et al 2006). It readily crosses the blood-brain barrier and peak cerebrospinal fluid levels are reached after $0.5-1.5$ hours.

The half-life of procarbazine is about 10 minutes and it is rapidly and extensively metabolized by the liver. Oxidation of procarbazine to azo-procarbazine occurs by microsomal cytotchrome $\mathrm{P} 450$ oxidoreductase, as well as by mitochondrial monoamine oxidase enzymatic conversion. Plasma levels of azo-procarbazine are notably higher than those of procarbazine itself. Azo-procarbazine is microsomally oxidized to azoxyderivatives. Of these, methylazoxy-procarbazine appears to be the major cytotoxic compound, showing several-fold greater cytotoxicity than procarbazine, azo-procarbazine, and benzyazoxyprocarbazine (Erikson et al 1989). Plasma levels of methylazoxy-procarbazine peak after about 1.5 hours and it has a half-life of around 1 hour.

About $70 \%$ of the dose is renally excreted in the first 24 hours; the major urinary metabolite is the biologically inactive $\mathrm{N}$-isopropylterphthalamic acid. The majority of the remainder is excreted via the respiratory system, with only minimal fecal excretion. Impaired renal or hepatic function can delay elimination of procarbazine and its active metabolites.

\section{Clinical efficacy Lymphomas \\ Hodgkin's lymphoma}

Hodgkin's lymphoma affects approximately 8000 new patients each year in the USA and around 1500 in the UK, occurring most commonly in young adults and in people who are more than 55 years old (Glaser and Jarrett 1996; ACS 2006). Although the management of the disease has improved considerably in recent years, around $20 \%$ to $30 \%$ of patients still experience disease progression or relapse.

Early studies reported overall response rates of 53\% to $69 \%$ with single agent procarbazine in patients with advanced 
Hodgkin's lymphoma (Brunner and Young 1965; Livingston and Carter 1970). However, these responses were generally very short-lived and there were few complete responses. In the early 1970s, procarbazine was used successfully in combination with three other drugs in the regimen known as MOPP (mechlorethamine [nitrogen mustard], Oncovin ${ }^{\circledR}$ [vincristine], procarbazine, and prednisone) in the treatment of advanced Hodgkin's lymphoma (DeVita et al 1970). A complete remission rate of $84 \%$ was attained, with relapsefree and overall survival rates of $66 \%$ and $48 \%$, respectively, after more than 20 years. Unfortunately, although the acute toxic effects of MOPP are manageable, it is associated with significant long-term toxicity. For example, more than $90 \%$ of men, and $80 \%-100 \%$ of women over 25 years old, become irreversibly infertile (Pazdur et al 2003). There is also a risk of secondary acute leukemia, which is frequently resistant to treatment and has an extremely poor prognosis ( $\mathrm{Ng}$ et al 2002). A number of MOPP-like regimens were developed in an attempt to reduce this toxicity. It would appear that procarbazine plays an important role in MOPP, as lower complete remission rates of shorter duration were reported with MOPP-like regimens that did not contain procarbazine (Nicholson et al 1970; Luce et al 1971).

ABVD (doxorubicin, bleomycin, vinblastine, and dacarbazine) subsequently became the regimen of choice for advanced disease after showing superior efficacy and less long-term toxicity than MOPP and MOPP hybrid regimens (Canellos et al 1992; Duggan et al 2003). The combination of MOPP plus epidoxirubicin, bleomycin, vinblastine, lomustine, doxorubicin, and vindesine (MOPPEBVCAD) has recently been suggested to be as effective as ABVD and more effective than the Stanford V schedule (doxorubicin, vinblastine, mechlorethamine, vincristine, bleomycin, etoposide, and prednisone) (Gobbi et al 2005, 2006). However, despite lower toxicity than normally expected with MOPP analogues, the incidence of second cancers remains somewhat higher than with ABVD. It is, nevertheless, a good option for patients who are not suitable for high-dose chemotherapy.

C-MOPP/ABV, a hybrid chemotherapy in which cyclophosphamide is substituted for mechlorethamine (which has been implicated as the cause of secondary malignancies) has shown beneficial effects (Montoto et al 2000). In 73 patients with advanced disease, 57 (78\%) achieved a complete response and 7 further patients who achieved partial response reached complete response after radiotherapy (Monoto et al 2000). The overall survival rate after 4 years was $92 \%$. In another study in which dacarbazine was added to the regimen (C-MOPP-ABVD), a response rate of $93 \%$ and a complete response rate of $84 \%$ was achieved amongst the 67 patients (Takenaka et al 2000).

A recent report of the long-term follow-up of 533 patients with advanced Hodgkin's lymphoma indicated that ABVPP (doxorubicin, bleomycin, vinblastine, procarbazine, and prednisone) was associated with a better survival probability than MOPP-ABV (Ferme et al 2006). The 10-year overall survival estimates were $90 \%$ for ABVPP, $78 \%$ for MOPP-ABV, $82 \%$ for MOPP-ABV and radiotherapy, and $77 \%$ for $\mathrm{ABVPP}$ and radiotherapy $(\mathrm{p}=0.03)$.

The German Hodgkin's Study Group developed a new combination regimen known as BEACOPP, based on a statistical model that considers tumor growth and chemotherapeutic effects (Loeffler et al 1998). The dose of procarbazine is $100 \mathrm{mg} / \mathrm{m}^{2}$ over 7 days, which is half that delivered over 14 days in the MOPP regimen. Both regimens are given on a 21-day cycle. In a randomized study, 1195 patients with newly diagnosed Hodgkin's lymphoma in an unfavorable stage (IIB, IIIA, IIIB, or IV) were randomized to receive eight cycles of COPP-ABVD (alternating cyclophosphamide, vincristine, procarbazine, and prednisone with doxorubicin, bleomycin, vinblastine, and dacarbazine), standard-dose BEACOPP, or increased-dose BEACOPP (Table 1), each followed by local radiotherapy when indicated (Diehl et al 2003). The outcome of treatment and five-year survival rates are shown in Table 2. The increased-dose BEACOPP regimen was significantly (pd $\leq 0.002)$ more effective than COPP-ABVD in terms of freedom from treatment failure ( $87 \%$ vs $69 \%$ ) and overall survival $(91 \%$ vs $83 \%)$ at 5 years, as well as early progression ( $2 \%$ vs $10 \%$ ). Standard-dose BEACOPP was also significantly $(p=0.04)$ more effective than COPP-ABVD for freedom from treatment failure at 5 years $(76 \%$ vs $69 \%)$. When the two BEACOPP regimens were compared with each other, the increased-dose regimen resulted in a significantly lower rate of early progression ( $2 \%$ vs $8 \%)$ and a significantly $(\mathrm{p}<0.001)$ greater freedom from treatment failure at 5 years $(87 \%$ vs $76 \%)$. Acute hematological effects were more common with the increased-dose BEACOPP regimen. The actuarial rate of secondary acute leukemia five years after diagnosis of Hodgkin's disease was also significantly $(\mathrm{p}=0.03)$ higher with increased-dose BEACOPP (2.5\%) compared with standard-dose $(0.6 \%)$, and COPP-ABVD $(0.4 \%)$.

The acute hematological toxicity of standard-dose and dose-intensified BEACOPP was investigated in 858 patients 
Table I COPP-ABVD, standard-dose BEACOPP, and increased-dose BEACOPP dosage regimens (Diehl et al 2003)

\begin{tabular}{|c|c|c|c|c|c|c|}
\hline \multirow{2}{*}{ BEACOPP ${ }^{b}$} & \multicolumn{2}{|c|}{ COPP-ABVD ${ }^{a}$} & \multicolumn{2}{|c|}{ Standard-dose BEACOPP } & \multicolumn{2}{|c|}{ Increased-dose } \\
\hline & $\begin{array}{l}\text { Single dose } \\
\left(\mathrm{mg} / \mathrm{m}^{2}\right)\end{array}$ & $\begin{array}{l}\text { Days } \\
\text { given }\end{array}$ & $\begin{array}{l}\text { Single dose } \\
\left(\mathrm{mg} / \mathrm{m}^{2}\right)\end{array}$ & $\begin{array}{l}\text { Days } \\
\text { given }\end{array}$ & $\begin{array}{l}\text { Single dose } \\
\left(\mathrm{mg} / \mathrm{m}^{2}\right)\end{array}$ & $\begin{array}{l}\text { Days } \\
\text { given }\end{array}$ \\
\hline Bleomycin & $10 \mathrm{iv}$ & 29 and 43 & $10 \mathrm{iv}$ & 8 & 10 iv & 8 \\
\hline Etoposide & - & - & $100 \mathrm{iv}$ & $1-3$ & $200 \mathrm{iv}$ & $1-3$ \\
\hline Doxorubicin & $25 \mathrm{iv}$ & 29 and 43 & 25 iv & 1 & $35 \mathrm{iv}$ & 1 \\
\hline Cyclophosphamide & $650 \mathrm{iv}$ & 1 and 8 & $650 \mathrm{iv}$ & 1 & $1200 \mathrm{iv}$ & 1 \\
\hline Vincristine & $\mathrm{I} .4 \mathrm{iv}$ & 1 and 8 & $\mathrm{I} .4 \mathrm{iv}$ & 8 & $1.4 \mathrm{iv}$ & 8 \\
\hline Procarbazine & 100 po & $\mathrm{I}-14$ & 100 po & $\mathrm{I}-7$ & 100 po & $\mathrm{I}-7$ \\
\hline Prednisone & 40 po & $\mathrm{I}-14$ & 40 po & $1-14$ & 40 po & $1-14$ \\
\hline Vinblastine & 6 iv & 29 and 43 & - & - & - & - \\
\hline Dacarbazine & $375 \mathrm{iv}$ & 29 and 43 & - & - & - & - \\
\hline
\end{tabular}

Note: ${ }^{a}$ regimen repeated on day 57; ${ }^{b}$ regimen repeated on day 22.

Abbreviations: BEACOPP, bleomycin, etoposide, doxorubicin, cyclophosphamide, vincristine, procarbazine, and prednisone; COPP-ABVD, alternating

cyclophosphamide, vincristine, procarbazine, and prednisone with doxorubicin, bleomycin, vinblastine, and dacarbazine; iv, intravenous; po, oral.

with advanced Hodgkin's lymphoma (Engel et al 2000). Dose-limiting toxicity occurred in $25 \%$ of cycles of doseintensified treatment, but severe thrombocytopenia and leukocytopenia were rare. Myelosuppression was the most important dose-limiting factor. The findings indicated that, despite some increase in hemotoxicity, moderate dose escalation was safe for most patients receiving granulocyte colony-stimulating factor (G-CSF) and standard supportive treatment.

BEACOPP has also been used successfully as salvage treatment in patients with early stage Hodgkin's lymphoma who relapsed after extended field radiotherapy. A retrospective analysis of 107 patients treated mainly with COPP-ABVD-like regimens $(\mathrm{n}=74)$ or BEACOPP $(\mathrm{n}=24)$ showed that BEACOPP was significantly $(\mathrm{p}=0.02)$ superior to the other regimens, with $100 \%$ of patients achieving complete remission compared with $85 \%$ of those given COPD-ABVD-like regimens, and $67 \%$ given other regimens including radiotherapy (Rüffer et al 2005). BEACOPP was also a significant $(p=0.03)$ prognostic factor for freedom from second failure.

In a study in children and adolescents with advanced disease, induction treatment with dose-intensified BEACOPP improved early tumor control, with a $45 \%$ response rate ( $>70 \%$ reduction in disease burden) after 2 cycles and a $72 \%$ response rate after 4 cycles (Kelly et al 2002).

\section{Non-Hodgkin's lymphoma}

Single-agent procarbazine is less active in non-Hodgkin's lymphoma than in Hodgkin's lymphoma, resulting in overall response rates of only around $36 \%-40 \%$ (Livingston and Carter 1970), although it has a role to play for patients who fail standard regimens or who cannot tolerate or refuse such treatment (Chaar et al 2006). Combination regimens containing procarbazine, such as MOPP and COPP (cyclophosphamide, Oncovin ${ }^{\circledR}$ [vincristine], procarbazine, and prednisone), have resulted in more beneficial effects, particularly in patients with advanced stage nodular mixed or lymphocytic poorly differentiated disease (Lowenbraun et al 1970; DeVita et al 1975; Longo et al 1984; Ezdinli et al 1985). The best response rates to regimens containing procarbazine are seen with intensive cyclic multi-drug combinations such as ProMACE-MOPP (procarbazine, methotrexate with leucovorin, doxorubicin, cyclophosphamide, and etoposide). In a randomized, controlled trial, 221 patients with diffuse intermediate- to high-grade non-Hodgkin's lymphoma received ProMACE-MOPP for 6 cycles or the newer generation more aggressive MACOP-B (methotrexate with leucovorin, doxorubicin, cyclophosphamide, vincristine, prednisone, and bleomycin) for 12 weeks (Sertoli et al 1994). There was no significant difference between the ProMACE-MOPP and MACOP-B groups in terms of complete remission rate $(49.1 \%$ and $52.3 \%$ ), three-year overall survival rate $(45.2 \%$ and $52.3 \%$ ), and three-year progression-free survival rate $(36.4 \%$ and $36.1 \%)$. Toxicity was also similar with both treatments. THPCOPBLM (pirarubicin, cyclophosphamide, vincristine, prednisone, bleomycin, and procarbazine) combined with GCSF in 42 patients with intermediate- or high-grade nonHodgkin's lymphoma resulted in a complete remission rate of $88 \%$ and a partial remission rate of $12 \%$ (Niitsu and Umeda 1998). The 3 -year survival rate was $72 \%$ and the 3 -year eventfree survival rate was $58 \%$. In 51 older patients (e $\geq 55$ years old) with intermediate-grade non-Hodgkin's lymphoma, COPBLAM-I (cyclophosphamide, vincristine, prednisone, 
Table 2 Outcome of treatment and five-year survival rates with COPP-ABVD, standard-dose BEACOPP, and increased-dose BEACOPP (Diehl et al 2003)

\begin{tabular}{llll}
\hline & $\begin{array}{l}\text { COPP-ABVD } \\
(\mathbf{n}=\mathbf{2 6 0})\end{array}$ & $\begin{array}{l}\text { Standard-dose BEACOPP } \\
(\mathbf{n}=\mathbf{4 6 9})\end{array}$ & $\begin{array}{l}\text { Increased-dose BEACOPP } \\
\mathbf{( n = 4 6 6 )}\end{array}$ \\
\hline Complete remission & $85(80-89)$ & $88(85-91)$ & $96(93-97)$ \\
Early progression & $10(7-15)$ & $8(5-10)$ & $2(1-4)^{* * \dagger}$ \\
$\begin{array}{l}\text { Freedom from treatment } \\
\text { failure at 5 years }\end{array}$ & $69(63-75)$ & $76(72-80)^{*}$ & $87(83-91)^{* * \dagger}$ \\
Overall survival at 5 years & $83(78-87)$ & $88(85-91)$ & $91(88-94)^{*}$ \\
\hline
\end{tabular}

Note: Values given as percentage ( $95 \%$ confidence interval); Early progression defined as progression during treatment or within 3 months after the end of treatment; ${ }^{*} \mathrm{p}<0.05$ vs COPP-ABVD; ${ }^{* *} \mathrm{p}<0.001$ vs COPP-ABVD; ${ }^{\dagger} \mathrm{p}<0.001$ vs standard-dose BEACOPP.

Abbreviations: BEACOPP, bleomycin, etoposide, doxorubicin, cyclophosphamide, vincristine, procarbazine, and prednisone; COP-ABVD, alternating cyclophosphamide, vincristine, procarbazine, and prednisone with doxorubicin, bleomycin, vinblastine, and dacarbazine.

bleomycin, doxorubicin, and procarbazine) resulted in a complete remission rate of $65 \%$ after a median follow-up of 2.4 years (Shpilberg et al 1994). The projected 3-year overall survival and progression-free survival rates were $58 \%$ and $77 \%$, respectively.

\section{Primary central nervous system lymphoma}

Primary central nervous system lymphoma is an aggressive non-Hodgkin's lymphoma arising in the brain, eyes, spinal cord, or leptomeninges. The incidence has increased markedly over the last two decades, both in patients with and without immunodeficiency (Corn et al 1997).

Although the use of radiotherapy prior to methotrexate improves survival in these patients, it is also associated with neurotoxic effects, such as severe cognitive dysfunction and ataxia (DeAngelis et al 1989). This is a particular problem because at least half of all patients are at least 60 years old at the time of diagnosis. To avoid this problem, a number of chemotherapeutic regimens have been investigated as sole therapy. Procarbazine crosses the blood-brain barrier and has therefore been included in some of these regimens.

In a study of 52 patients treated with 5 cycles of highdose methotrexate, procarbazine, and vincristine, with or without whole-brain radiotherapy, the objective response rate was $90 \%$ and the median survival 60 months (Abrey et al 2000). The median survival was similar amongst the older patients ( $>60$ years old), irrespective of whether or not they received radiotherapy, but late neurotoxicity was significantly $(p=0.0004)$ more common in those who received radiotherapy. Another study, conducted by the European Organisation for Research and Treatment of Cancer (EORTC) Brain Tumour Group in 50 elderly patients (median age 72 years), investigated the use of high-dose methotrexate, lomustine, procarbazine, methylprednisolone, and intrathecal chemotherapy with methotrexate and cytarabine (Hoang-Xuan et al 2003). The objective response rate was $48 \%$ ( $42 \%$ complete and $6 \%$ partial responses); the median duration of the response in the patients with complete response was 27 months; the median survival was 14.3 months; and the one-year progression-free survival was $40 \%$. The majority of patients had improved or preserved cognitive function and Karnofsky performance scores (KPS) until relapse. A decline in cognitive function and KPS attributed to late neurotoxicity occurred in only $8 \%$ and $12 \%$ of patients, respectively. In the long-term responders who were in remission 2 years after the start of therapy, only one patient showed a decline in cognitive function or performance status due to delayed neurotoxicity. This outcome was superior to that expected with radiotherapy alone in elderly patients and considerably reduced the risk of delayed neurotoxicity.

Blood-brain barrier disruption (BBBD) results in enhanced chemotherapy delivery. In 74 patients who underwent BBBD preceded by systemic cyclophosphamide and followed by intra-arterial methotrexate in combination with procarbazine and dexamethasone, a complete response

Table 3 PCV and intensified PCV regimens

\begin{tabular}{lllll}
\hline & PCV & Intensified PCV & Days of administration & Frequency \\
\hline Procarbazine & $60 \mathrm{mg} / \mathrm{m}^{2}$ po & $75 \mathrm{mg} / \mathrm{m}^{2}$ po & $8-21$ & Every 6-8 weeks \\
Lomustine & $110 \mathrm{mg} / \mathrm{m}^{2}$ po & $130 \mathrm{mg} / \mathrm{m}^{2}$ po & 8 and 29 & \\
Vincristine & $1.4 \mathrm{mg} / \mathrm{m}^{2}$ iv & $1.4 \mathrm{mg} / \mathrm{m}^{2}$ iv & 1 & \\
\hline
\end{tabular}

Abbreviations: $\mathrm{PCV}$, procarbazine, lomustine (CCNU), vincristine. 
was achieved in $65 \%(\mathrm{n}=48)$ and a partial response in $19 \%$ $(\mathrm{n}=14)$ (McAllister et al 2000). Amongst the 36 patients who continued to show a complete response after one year, none had any evidence of cognitive loss.

Procarbazine has also been used as part of the PCV (procarbazine, $\mathrm{CCNU}$, and vincristine) regimen. Although the prognosis at relapse is often poor for patients suffering from primary central nervous system lymphoma, treatment of 7 relapsed patients with PCV resulted in a complete response in 4 and a partial response in 2 (Herrlinger et al 2000).

\section{Brain tumors-glioma/astrocytoma}

Gliomas comprise about half of all primary brain tumors and include astrocytomas, glioblastomas, and oligodendrogliomas. The dosage details for the PCV and intensified PCV regimens are shown in Table 3. A summary of the main clinical trials into the use of singleagent procarbazine and procarbazine-containing regimens in patients with glioma is provided in Table 4.

Interest in the use of procarbazine emerged around 1990 when data from the Northern California Oncology Group was reanalyzed to compare the effects of carmustine (BCNU) with that of PCV following radiation/oral hydroxyurea in patients with glioblastoma multiforme or other anaplastic gliomas (Levin et al 1990). PCV treatment resulted in longer survival and time to tumor progression than BCNU, with the difference achieving statistical significance in the group of patients with anaplastic gliomas.

In a randomized study of 171 patients with grade III/IV astrocytoma given PCV, with or without radiotherapy, the median time to progression was 21 weeks and the median survival time was 53 weeks (Sandberg-Wollheim et al 1991). Overall, $18 \%$ of patients survived for at least two years. Survival analysis showed that the best responses were achieved in patients less than 50 years old who received both PCV and radiotherapy (median time to progression and survival time of 81 and 124 weeks, respectively).

A durable response to PCV was reported by Kim and colleagues (1996) in 32 patients with grade III/IV oligoastrocytoma or anaplastic oligodendroglioma. The PCV therapy was administered every 6 weeks for a total of at least 124 cycles. The median duration of follow-up review from the start of chemotherapy was 19.3 months. Grade 3 or 4 hematological toxicity was experienced by nine (31\%) of 29 patients. Ten patients had delayed treatment due to treatment-related toxicities (34.5\%). Ninety-one percent of the 32 patients responded to the therapy. These included 10 patients with a complete response and 19 with a partial response. The median time to progression was 15.4 months for all patients and 23.2 months for those with Grade III tumors. The median time to progression for patients with Grade III oligoastrocytomas was 13.8 months; for those with Grade IV oligoastrocytoma it was 12.4 months, and for those with anaplastic oligodendrogliomas it was 63.4 months ( $p=0.0348)$. These patients survived a median of 49.8 months, 16 months, and 76 or more months, respectively, from the start of chemotherapy $(\mathrm{p}=0.0154)$, thus showing that PCV therapy provides durable responses in patients with Grade III or IV oligoastrocytomas.

A number of other studies found no marked beneficial effects of PCV therapy on survival (Prados et al 1999; Kappelle et al 2001; MRC 2001) and so there has been an increasing tendency to use procarbazine as a salvage option. However, little data are available in the literature regarding this strategy (Rodriguez et al 1989; Newton et al 1990; Brandes et al 1999; Newlands et al 2003). Response rates of $15 \%$ to $25 \%$ were reported with single-agent procarbazine in patients with recurrent glioblastoma or anaplastic astrocytoma (Rodriguez et al 1989; Newton et al 1990). An overall response rate of $30 \%$ was achieved with combined procarbazine and tamoxifen in patients with high-grade gliomas that recurred after surgery and radiotherapy (Brandes et al 1999). Compared with patients suffering from glioblastoma, those with anaplastic astrocytoma had a significantly longer median time to progression (33 vs 13 weeks) and median survival time (57 vs 27 weeks).

Recent results of adjuvant PCV in newly diagnosed patients with anaplastic oligodendroglioma are extremely encouraging (Buckner et al 2003; Stege et al 2005; van den Bent et al 2005, 2006; Yamamoto et al 2005). PCV resulted in tumor regression in a meaningful proportion of newly diagnosed low-grade oligodendroglioma, with or without loss of 1p/19q. In a phase III EORTC study (van den Bent et al 2005, 2006), 6 cycles of adjuvant PCV following radiotherapy in 368 patients with highly anaplastic oligodendroglioma prolonged the progression-free survival from 13 to 23 months compared with radiotherapy alone $(\mathrm{p}=0.0018)$ (Figure 1). Overall survival was also longer with adjuvant PCV, but the difference did not reach statistical significance (Figure 1). The percentage of patients with progression-free survival after 2 years and overall survival after 5 years also showed advantages for adjuvant PCV treatment (Figure 2). Recent evidence suggests that PCV may also be beneficial in patients with symptomatic low-grade oligodendroglioma (Catenoix et al 2006).

Beneficial effects were reported with 6-thioguanine, procarbazine, dibromodulcitol, lomustine, and vincristine 


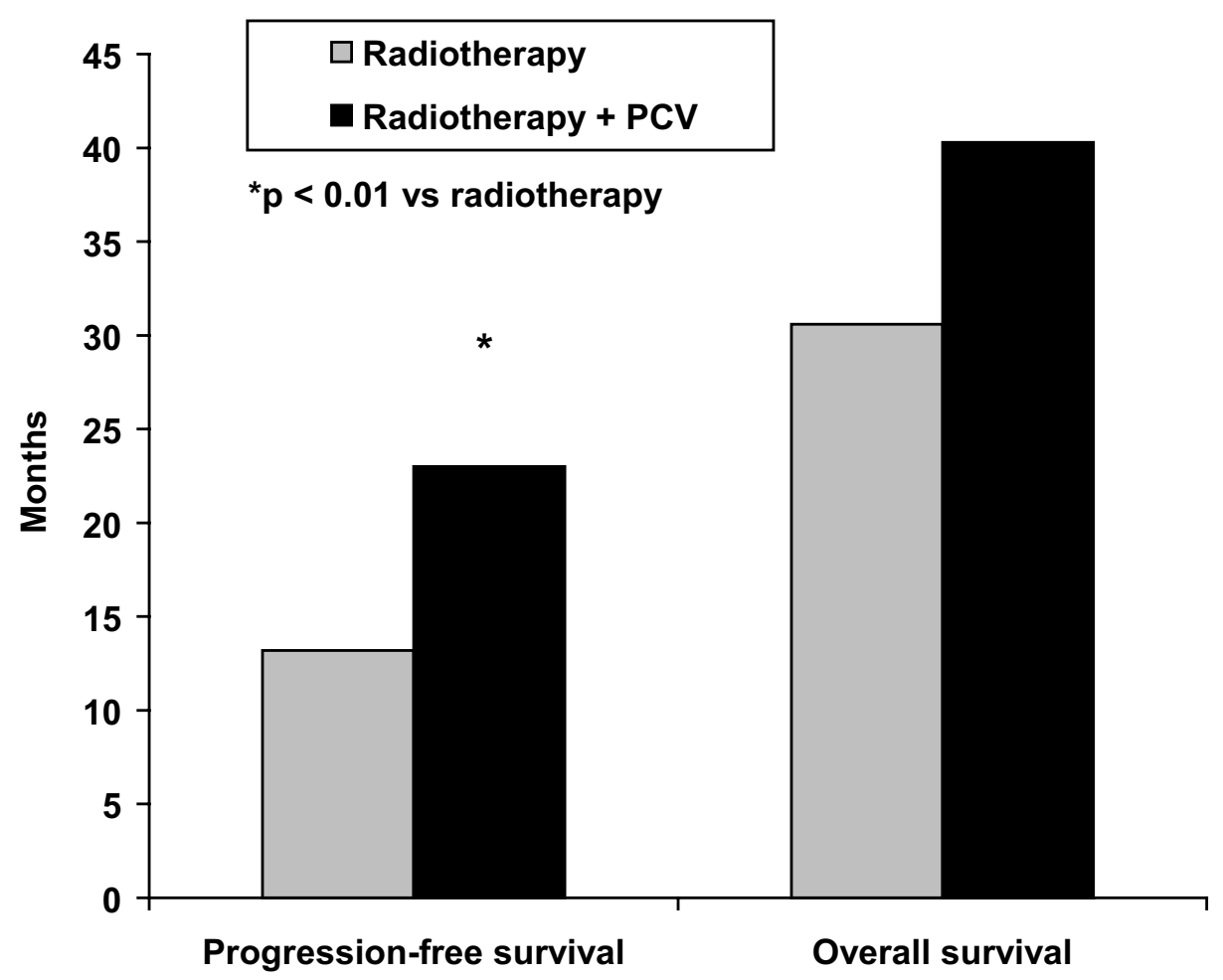

Figure I Median progression-free survival and overall survival in patients with newly diagnosed anaplastic oligodendroglioma treated with radiotherapy alone or radiotherapy plus PCV (procarbazine, CCNU, and vincristine) (van den Bent et al 2006).

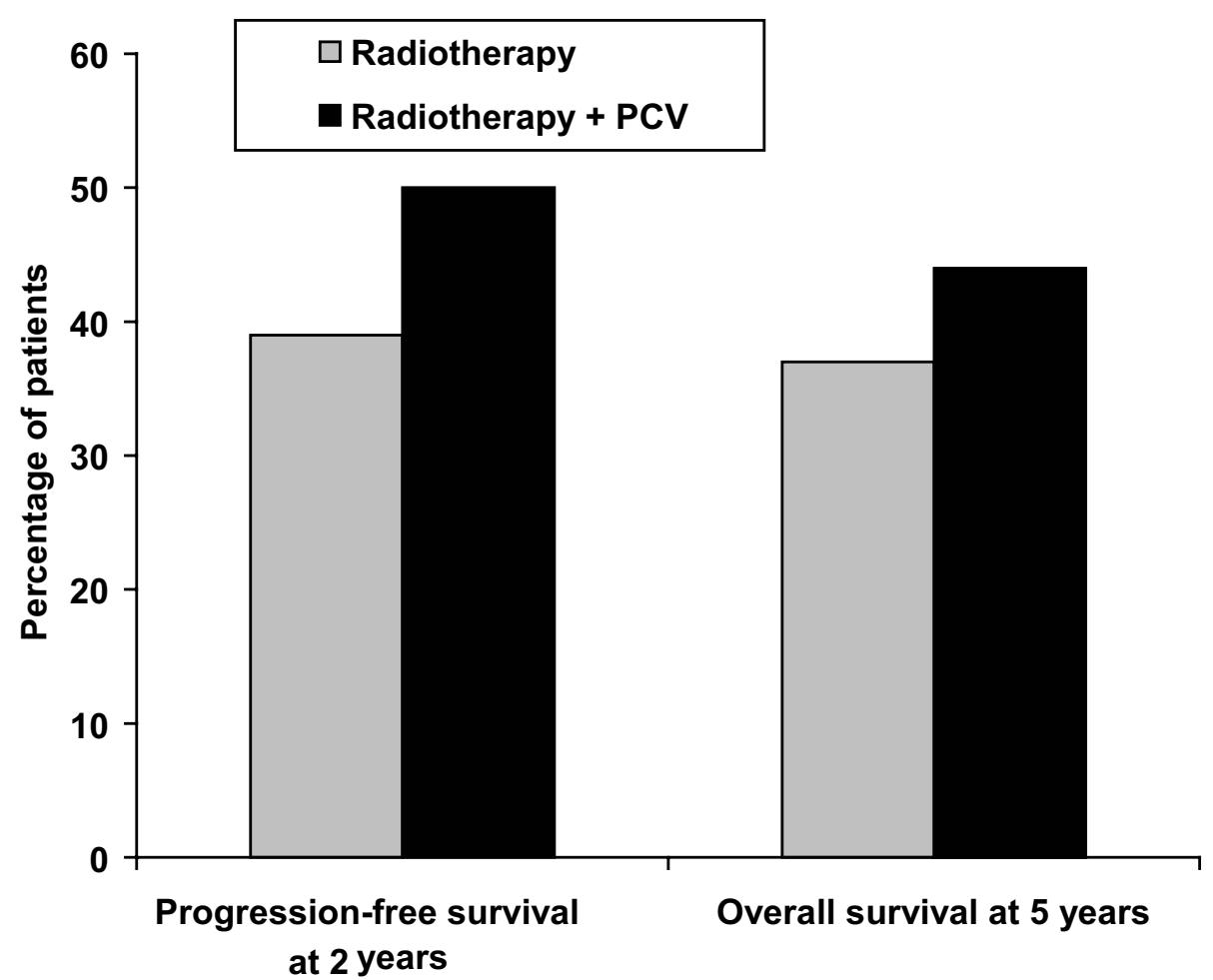

Figure 2 Percentage of patients with progression-free survival after 2 years and overall survival after 5 years in patients with newly diagnosed anaplastic oligodendroglioma treated with radiotherapy alone or radiotherapy plus PCV (procarbazine, CCNU, and vincristine) (van den Bent et al 2006). 


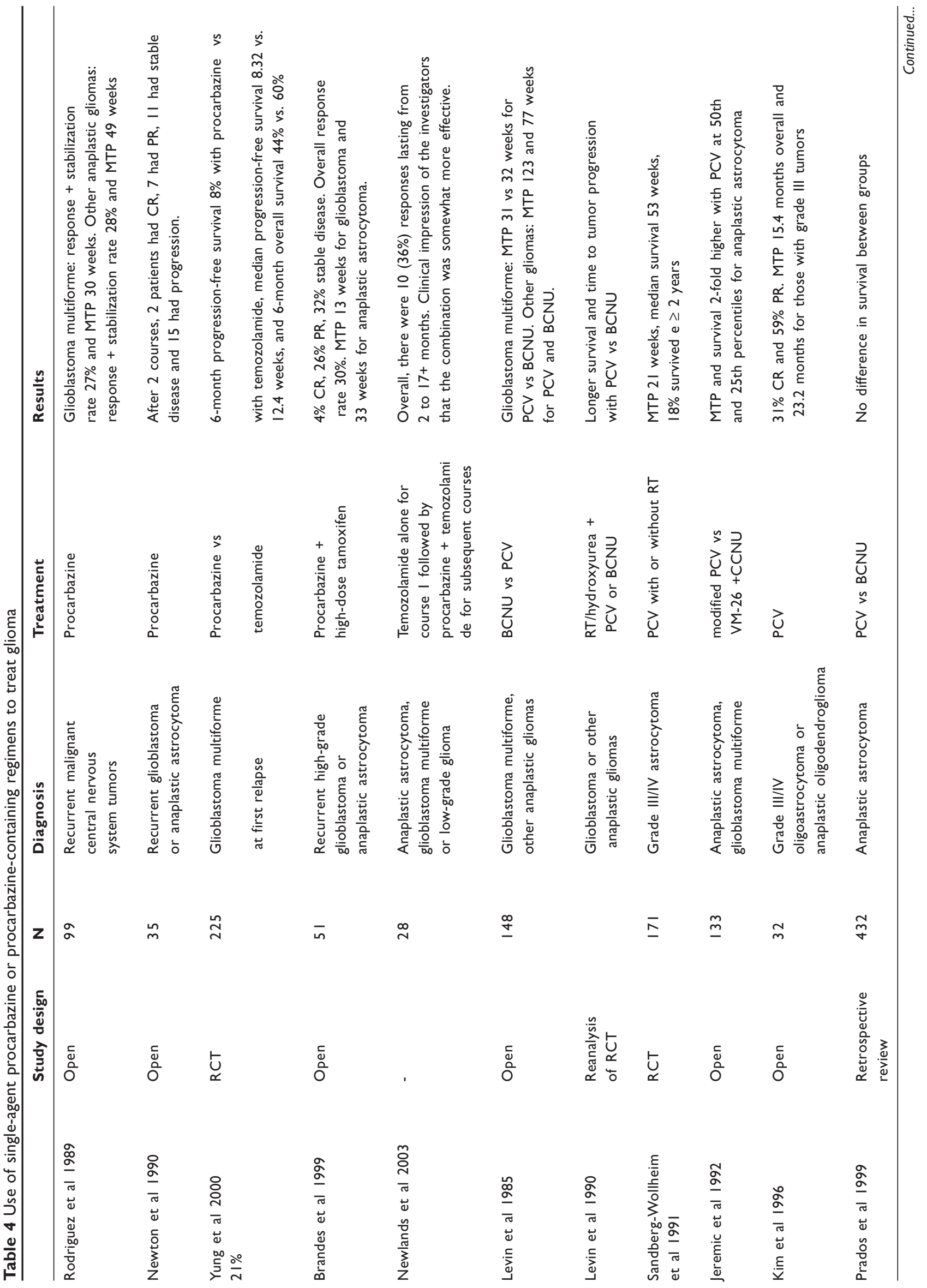




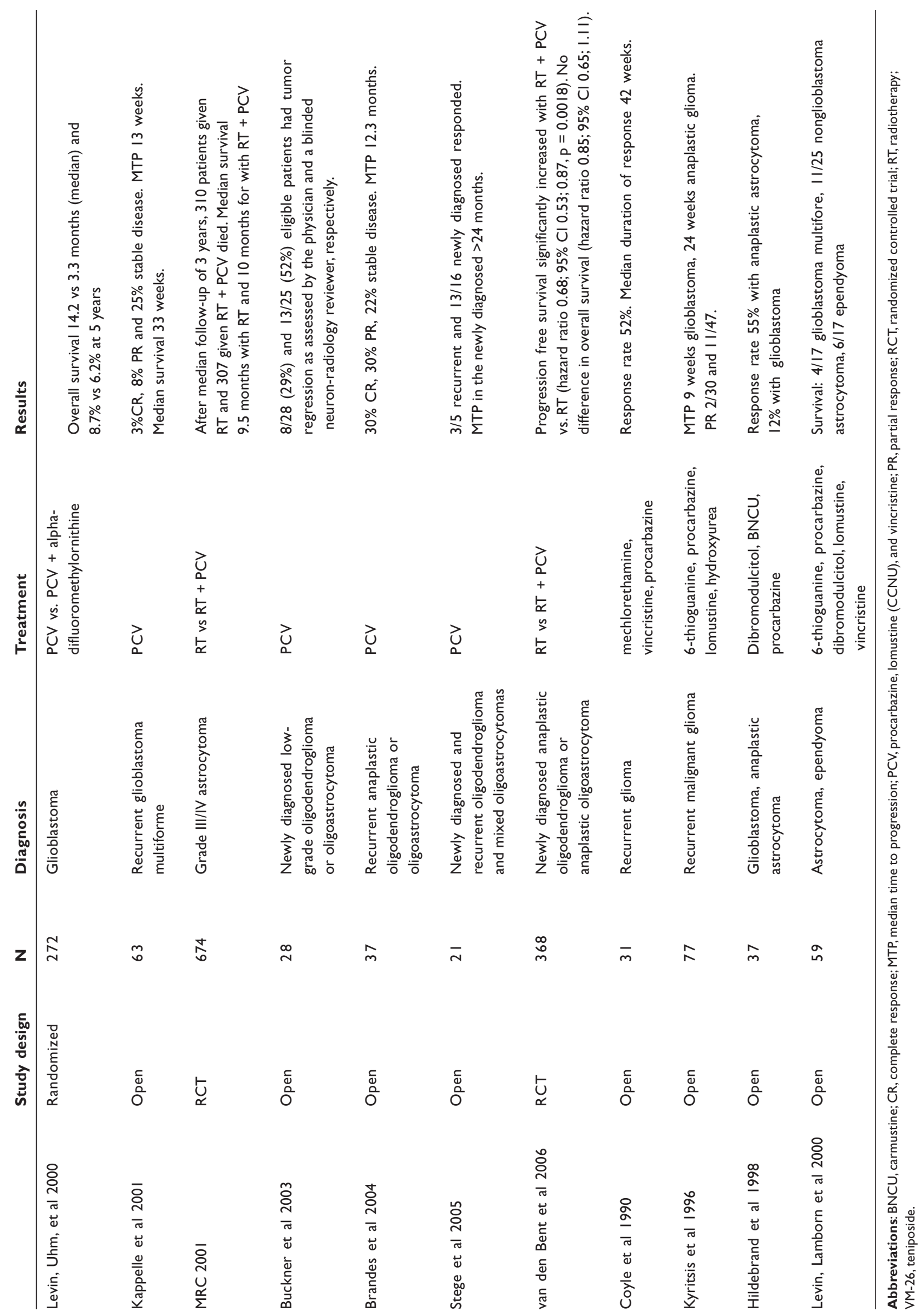


(TPDCV) in 42 children and young people with malignant astrocytomas (Levin et al 2000). Of the 17 patients with glioblastoma multiforme, 13 died, the median time to progression was 49 weeks and median survival was 85 weeks. Amongst the remaining 25 patients with nonglioblastoma malignant astrocytoma, 14 died and the median time to progression was 224 weeks. The median survival was not reached in this group after a median follow-up of 494 weeks. The BBSFOP protocol, which comprises seven cycles of three drug pairs (carboplatin-procarbazine, cisplatin-etoposide and vincristine-cyclopho-sphamide), was used as first line treatment to avoid radiotherapy in 21 children ( $<5$ years old) with highgrade glioma (Dufour et al 2006). The 5-year progressionfree survival was $35 \%$ and the 5-year overall survival was $59 \%$, with a median follow-up of 5.2 years. Of the 12 children still alive, 10 have not required radiotherapy.

Procarbazine, nimustine, and vincristine given in combination with hyperfractionated radiotherapy resulted in an objective response in 3 out of 8 patients with high-grade glioma (Ogawa et al 2006). The median time to progression was 10.7 months and the median survival time of all patients was 15.0 months. The treatment was safe and well tolerated.

Recent evidence indicates that enzyme-inducing antiseizure medications have no effect on the pharmacokinetics of procarbazine in patients with glioma, but that there may be an increased risk of hepatic dysfunction with this drug combination (Grossman et al 2006).

\section{Safety and tolerability}

Gastrointestinal disturbances (eg, nausea and vomiting), myelosuppression (eg, leukopenia, anemia, and thrombopenia) and central nervous system effects (eg, headache, depression, nervousness, and insomnia) are the most common side-effects seen with procarbazine, followed by neuromuscular problems such as tremor, weakness, and parasthesia. Procarbazine has also been associated with secondary nonlymphoid malignancies, including acute leukemia and solid tumors such as lung cancer, when used in combination therapy. Azoospermia and adverse effects on female gonadal function may also be a problem. Rare side-effects include hemolysis in patients with glucose 6-phosphate dehydrogenase deficiency, hypersensitivity reactions, eosinophilia, and pulmonary infiltrates.

Since procarbazine is a weak monoamine oxidase inhibitor, there is a possibility of interactions with food containing high levels of tyramine (eg, beer, cheese, wine, bananas) and with certain drugs such as sympathomimetics and decongestants. Possible effects include hypertensive crisis, intracranial bleeding, and headache. Procarbazine can also interact with other medications. For example, central nervous system depressants (eg, anesthetics, barbiturates, narcotic analgesics), drugs with anticholinergic effects (eg, tricyclic antidepressants), and antihypertensive agents should all be used with caution and in reduced dosage in patients taking procarbazine due to possible potentiation effects. Alcohol should also be avoided as disulfiram-like effects such as sweating and facial flushing may occur.

\section{Discussion and place in therapy}

Procarbazine is a long established anticancer medicine. Although there is renewed interest in this agent, it is worthwhile to remember the many patient-years of experience in many different oncological indications which exist; few 'new' cancer therapies have the benefit of such experience.

Although there was a long period in which interest in procarbazine waned due to safety concerns and the development of other cancer treatments, interest is currently re-emerging, mainly in combination with other agents for the treatment of Hodgkin's lymphoma and gliomas, for which procarbazine activity is high. Some of the early combination therapies which used procarbazine (such as MOPP) were effective, but their use was limited by late toxicity. The new generation of procarbazine-containing combination therapies have been optimized to maximize efficacy and minimize the late-toxic effects seen with the first combinations.

Procarbazine looks likely to continue in the anticancer armamentarium for some time to come and, as a component of the BEACOPP combination, become part of the proposed 'gold standard' treatment for Hodgkin's lymphoma (Massoud et al 2004). Recent results of adjuvant PCV in newly diagnosed patients with anaplastic oligodendroglioma are also extremely encouraging. Despite its long history, procarbazine is still an important drug in the treatment of Hodgkin's lymphoma and gliomas.

\section{References}

Abrey LE, Yahalom J, DeAngelis LM. 2000. Treatment for primary CNS lymphoma: the next step. J Clin Oncol, 18:3144-50.

[ACS] American Cancer Society. 2006. Cancer facts and figures 2006 [online]. Accessed on 28 December 2006. URL: http://www.cancer.org/ downloads/STT/CAFF2006PWSecured.pdf.

Berneis K, Kofler M, Bollag W, et al. 1963. The degradation of DNA by a new tumor inhibiting compound. Experientia, 19:132-3.

Bollag W, Grunberg E. 1963. The degradation of deoxyribonucleic acid by new tumour inhibiting compounds: the intermediate formation of hydrogen peroxide. Experientia, 19:130-1. 
Brandes AA, Ermani M, Turazzi S, et al. 1999. Procarbazine and highdose tamoxifen as a second-line regimen in recurrent high-grade gliomas: a phase II study. J Clin Oncol, 17:645-50.

Brandes AA, Tosoni A, Vastola F, et al. 2004. Efficacy and feasibility of standard procarbazine, lomustine, and vincristine chemotherapy in anaplastic oligodendroglioma and oligoastrocytoma recurrent after radiotherapy. A Phase II study. Cancer, 101:2079-85.

Brunner KW, Young CW. 1965. A methylhydrazine derivative in Hodgkin's disease and other malignant neoplasms. Therapeutic and toxic effects studied in 51 patients. Ann Intern Med, 63:69-86.

Buckner JC, Gesme D Jr, O'Fallon JR, et al. 2003. Phase II trial of procarbazine, lomustine, and vincristine as initial therapy for patients with low-grade oligodendroglioma or oligoastrocytoma: efficacy and associations with chromosomal abnormalities. J Clin Oncol, 21:251-5.

Canellos GP, Anderson JR, Propert KJ, et al. 1992. Chemotherapy of advanced Hodgkin's disease with MOPP, ABVD, MOPP alternating with ABVD. NEngl J Med, 327:1478-84.

Catenoix H, Honnorat J, Cartalat-Carel S, et al. 2006. [Long-term outcome in patients with symptomatic low-grade oligodendrogliomatous tumors treated by cytotoxic agents.] Rev Neurol, 162:1069-75.

Chaar BT, Salem P, Petruska PJ. 2006. Procarbazine for non-Hodgkin's lymphoma. Leukemia Lymphoma, 47:637-40.

Chryssanthou CP, Wallach RC, Atchison M. 1983. Meiotic chromosomal changes and sterility produced by nitrogen mustard and procarbazine in mice. Fertil Steril, 39:97-102.

Corn BW, Marcus SM, Topham A, et al. 1997. Will primary central nervous system lymphoma be the most frequent brain tumor diagnosed in the year 2000? Cancer, 79:2409-13.

Coyle T, Baptista J, Winfield J, et al. 1990. Mechlorethamine, vincristine and procarbazine chemotherapy for recurrent high-grade glioma in adults: a phase II study. J Clin Oncol, 8:2014-18.

DeAngelis LM, Delattre JY, Posner JB. 1989. Radiation-induced dementia in patients cured of brain metastases. Neurology, 39:789-96.

DeVita VT Jr, Canellos GP, Chabner B, et al. 1975. Advanced diffuse histiocytic lymphoma, a potentially curable disease. Lancet, 1:248-50.

Devita VT Jr, Serpick AA, Carbone PP. 1970. Combination chemotherapy in the treatment of advanced Hodgkin's disease. Ann Intern Med, 73:881-95.

Diehl V, Franklin J, Pfreundschuh M, et al. 2003. Standard and increaseddose BEACOPP chemotherapy compared with COPP-ABVD for advanced Hodgkin's disease. $N$ Engl J Med, 348:2386-95.

Dufour C, Grill J, Lellouch-Tubiana A, et al. 2006. High-grade glioma in children under 5 years of age: A chemotherapy only approach with the BBSFOP protocol. Eur J Cancer, 42:2939-45.

Duggan DB, Petroni GR, Johnson JL, et al. 2003. Randomized comparison of $\mathrm{ABVD}$ and MOPP/ABV hybrid for the treatment of advanced Hodgkin's disease: report of an Intergroup trial. J Clin Oncol, 21:607-14.

Engel C, Loeffler M, Schmitz H, et al. 2000. Acute hematologic toxicity and practicability of dose-intensified BEACOPP chemotherapy for advanced stage Hodgkin's disease. German Hodgkin's Lymphoma Study Group (GHSG). Ann Oncol, 11:1105-14.

Erikson JM, Tweedie DJ, Ducore JM, et al. 1989. Cytotoxicity and DNA damage caused by the azoxy metabolites of procarbazine in L1210 tumor cells. Cancer Res, 49:127-33.

Ezdinli EZ, Anderson JR, Melvin F, et al. 1985. Moderate versus aggressive chemotherapy of nodular lymphocytic poorly differentiated lymphoma. J Clin Oncol, 3:769-75.

Ferme C, Mounier N, Casasnovas O, et al. 2006. Long-term results and competing risk analysis of the $\mathrm{H} 89$ trial in patients with advanced-stage Hodgkin lymphoma: a study by the Groupe d'Etude des Lymphomes de l'Adulte (GELA). Blood, 107:4636-42.

Friedman HS. 2001. Alkylating agents in cancer chemotherapy and biotherapy. Chabner BA, Longo DL (eds). 4th edition Philadelphia; Lippincott Williams \& Wilkins, p 415.
Friedman HS, Johnson SP, Dong Q, et al. 1997. Methylator resistance mediated by mismatch repair deficiency in a glioblastoma multiforme xenograft. Cancer Res, 57:2933-6.

Glaser SL, Jarrett RF. 1996. The epidemiology of Hodgkin's disease. Baillieres Clin Haematol, 9:401-6.

Gobbi PG, Broglia C, Levis A, et al. 2006. MOPPEBVCAD chemotherapy with limited and conditioned radiotherapy in advanced Hodgkin's lymphoma: 10-year results, late toxicity, and second tumors. Clin Cancer Res, 12:529-35.

Gobbi PG, Levis A, Chisesi T, et al. 2005. ABVD versus modified Stanford $\mathrm{V}$ versus MOPPEBVCAD with optional and limited radiotherapy in intermediate- and advanced-stage Hodgkin's lymphoma: final results of a multicenter randomized trial by the Intergruppo Italiano Linfomi. $J$ Clin Oncol, 23:9198-207.

Grossman SA, Carson KA, Batchelor TT, et al. 2006. The effect of enzymeinducing antiseizure drugs on the pharmacokinetics and tolerability of procarbazine hydrochloride. Clin Cancer Res, 12:5174-81.

Herrlinger U, Brugger W, Bamberg M, et al. 2000. PCV salvage chemotherapy for recurrent primary CNS lymphoma. Neurology, $54: 1707-8$.

Hildebrand J, de Witte O, Sahmoud T. 1998. Response of recurrent glioblastoma and anaplastic astrocytoma to dibromodulcitol, BCNU and procarbazine - a phase II study. J Neurooncol, 37:155-60.

Hoang-Xuan K, Taillandier L, Chinot O, et al. 2003. Chemotherapy alone as initial treatment for primary CNS lymphoma in patients older than 60 years: a multicenter phase II study (26952) of the European Organization for Research and Treatment of Cancer Brain Tumor Group. J Clin Oncol, 21:2726-31.

Jeremic B, Jovanovic D, Djuric LJ, et al. 1992. Advantage of postradiotherapy chemotherapy with $\mathrm{CCNU}$, procarbazine and vincristine (mPCV) over chemotherapy with VM-26 and CCNU for malignant gliomas. $J$ Chemother, 4:123-6.

Kappelle AC, Postma TJ, Taphoorn MJ, et al. 2001. PCV chemotherapy for recurrent glioblastoma multiforme. Neurol, 56:118-20.

Kelly KM, Hutchinson RJ, Sposto R, et al. 2002. Feasibility of upfront doseintensive chemotherapy in children with advanced-stage Hodgkin's lymphoma: preliminary results from the Children's Cancer Group Study CCG-59704. Ann Oncol, 13 (Suppl 1):107-11.

Kenis Y, De Smedt J, Tagnon HJ. 1966. The action of Natulan in 94 cases of solid tumors. Eur J Cancer, 2:51-7.

Kim L, Hochberg FH, Thornton AF, et al. 1996. Procarbazine, lomustine, and vincristine $(\mathrm{PCV})$ chemotherapy for grade III and grade IV oligoastrocytomas. J Neurosur, 85:602-7.

Kyritsis AP, Yung WK, Jaeckle KA, et al. 1996. Combination of 6thioguanine, procarbazine, lomustine and hydroxyurea for patients with recurrent malignant gliomas. Neurosurgery, 39:921-6.

Levin VA, Lamborn K, Wara W, et al. 2000. Phase II study of 6-thioguanine, procarbazine, dibromodulcitol, lomustine and vincristine chemotherapy with radiotherapy for treating malignant glioma in children. Neuro-oncol, 2:22-8.

Levin VA, Silver P, Hannigan J, et al. 1990. Superiority of postradiotherapy adjuvant chemotherapy with $\mathrm{CCNU}$, procarbazine, and vincristine (PCV) over BCNU for anaplastic gliomas. NCOG 6G61 final report. Int J Radiat Oncol Biol Phys, 18:321-4.

Levin VA, Uhm JH, Jaeckle KA, et al. 2000. Phase III randomized study of postradiotherapy chemotherapy with alpha-difluoromethylornithineprocarbazine, $\mathrm{N}$-(2-chloroethyl)-N'-cyclohexyl-N-nitrosurea, vincristine (DFMO-PCV) versus PCV for glioblastoma multiforme. Clin Cancer Res, 6:3878-84.

Levin VA, Wara WM, Davis RL, et al. 1985. Phase III comparison of $\mathrm{BCNU}$ and the combination of procarbazine, $\mathrm{CCNU}$ and vincristine administered after radiotherapy with hydroxyurea for malignant gliomas. J Neurosurg, 63:218-23.

Livingston RB, Carter SK. 1970. Single agents in cancer chemotherapy. New York: IFI/Plenum Data Corp. p 318-36. 
Loeffler M, Hasenclever D, Diehl V. 1998. Model based development of the BEACOPP regimen for advanced Hodgkin's disease. German Hodgkin's Lymphoma Study Group. Ann Oncol, 9:73-8.

Longo DL, Young RC, Hubbard SM, et al. 1984. Prolonged initial remission in patients with nodular mixed lymphoma. Ann Intern Med, 100:651-6.

Lowenbraun S, DeVita VT, Serpick AA. 1970. Combination chemotherapy with nitrogen mustard, vincristine, procarbazine, and prednisone in lymphosarcoma and reticulum cell sarcoma. Cancer, 25:1018-25.

Luce JK, Gamble JF, Wilson HE, et al. 1971. Combined cyclophosphamide vincristine, and prednisone therapy of malignant lymphoma. Cancer, 28:306-17.

McAllister LD, Doolittle ND, Guastadisegni PE, et al. 2000. Cognitive outcomes and long-term follow-up results after enhanced chemotherapy delivery for primary central nervous system lymphoma. Neurosurgery, 46:51-61.

Malek FA, Moritz KU, Fanghanel J. 2003. Effects of prenatal procarbazine administration on intrauterine development in rats. Ann Anat, 185:117-19.

Martin H, Schubert JC. 1966. Cytostatic treatment of polycythemia vera with a methylbenzylhydrazine derivative. Dtsch Med Wochenschr, 91:55-7.

Massoud M, Armand JP, Ribrag V. 2004. Procarbazine in haematology: an old drug with a new life? Eur J Cancer, 40:1924-7.

Montoto S, Camos M, Lopez-Guillermo A, et al. 2000. Hybrid chemotherapy consisting of cyclophosphamide, vincristine, procarbazine, prednisone, doxorubicin, bleomycin and vinblastine (C-MOPP/ABV) as first-line treatment for patients with advanced Hodgkin disease. Cancer, 88:2142-8.

[MRC] Medical Research Council Brain Tumor Working Party. 2001. Randomized trial of procarbazine, lomustine, and vincristine in the adjuvant treatment of high-grade astrocytoma: a Medical Research Council trial. J Clin Oncol, 19:509-18.

Newell D, Gescher A, Harland S, et al. 1987. N-methyl antitumor agents: a distinct class of anticancer drugs? Cancer Chemother Pharmacol, 19:91-102.

Newlands ES, Foster T, Zaknoen S. 2003. Phase I study of temozolamide (TMZ) combined with procarbazine (PCB) in patients with gliomas. Br J Cancer, 89:248-51.

Newton HB, Junck L, Bromberg J, et al. 1990. Procarbazine chemotherapy in the treatment of recurrent malignant astrocytomas after radiation and nitrosourea failure. Neurol, 40:1743-6.

$\mathrm{Ng} \mathrm{AK}$, Bernardo MV, Weller E, et al. 2002. Second malignancy after Hodgkin disease treated with radiation therapy with or without chemotherapy: long-term risks and risk factors. Blood, 100:1989-96.

Nicholson WM, Beard ME, Crowther D, et al. 1970. Combination chemotherapy in generalized Hodgkin's disease. $\mathrm{Br}$ Med J, 3:7-10.

Niitsu N, Umeda M. 1998. Biweekly THP-COPBLM (pirarubicin, cyclophosphamide, vincristine, prednisone, bleomycin and procarbazine) regimen combined with granulocyte colony-stimulating factor (G-CSF) for intermediate- and high-grade non-Hodgkins's lymphoma. Leukemia, $12: 1457-60$

Pazdur R, Coia LR, Hoskins WJ, et al. 2003. Cancer management: a multidisciplinary approach. New York: The Oncology Group.

Pegg AE. 1990. Mammalian O6-alkylguanine-DNA alkyltransferase: regulation and importance in response to alkylating carcinogenic and therapeutic agents. Cancer Res, 50:6119-29.

Prados MD, Scott C, Curran WJ, Jr, et al. 1999. Procarbazine, lomustine, and vincristine (PCV) chemotherapy for anaplastic astrocytoma: A retrospective review of radiation therapy oncology group protocols comparing survival with carmustine or PCV adjuvant chemotherapy. J Clin Oncol, 17:3389-95.
Preiss R, Baumann F, Regenthal R, et al. 2006. Plasma kinetics of procarbazine and azo-procarbazine in humans. Anti-Cancer Drugs, 17:75-80.

Rodriguez LA, Prados M, Silver P, et al. 1989. Reevaluation of procarbazine for the treatment of recurrent malignant central nervous system tumors. Cancer, 64:2420-3.

Rüffer JU, Ballova V, Glossmann J, et al. 2005. BEACOPP and COPP/ABVD as salvage treatment after primary extended field radiation therapy of early stage Hodgkins disease - results of the German Hodgkin Study Group. Leuk Lymphoma, 46:1561-7.

Rutinshauser A, Bollag W. 1963. Cytological investigations of a new class of cytotoxic agents methylhydrazine derivatives. Experientia, 19:131-2.

Sandberg-Wollheim M, Malmstrom P, Stromblad L, et al. 1991. A randomized study of chemotherapy with procarbazine, vincristine, and lomustine with and without radiation therapy for astrocytoma grades 3 and/or 4. Cancer, 68:22-9.

Sertoli MR, Santini G, Chisesi T, et al. 1994. MACOP-B versus ProMACEMOPP in the treatment of advanced diffuse non-Hodgkin's lymphoma: results of a prospective randomized trial by the non-Hodgkin's Lymphoma Cooperative Study Group. J Clin Oncol, 12:1366-74.

Shpilberg O, Shiff J, Chetrit A, et al. 1994. The cyclophosphamide, vincristine, prednisone, bleomycin, doxorubicin, and procarbazine (COPBLAM-I) regimen for intermediate-grade non-Hodgkin's lymphoma. Long term follow-up in 51 patients. Cancer, 74:3029-33.

Stege EM, Kros JM, de Bruin HG, et al. 2005. Successful treatment of lowgrade oligodendroglial tumors with a chemotherapy regimen of procarbazine, lomustine, and vincristine. Cancer, 103:802-9.

Supko JG, He X, Whitney C, et al. 2002. Pharmacokinetics of procarbazine and metabolites involved in its bioactivation in patients with recurrent anaplastic astrocytoma [abstract]. Proc Am Soc Clin Oncol, 21:2086.

Takenaka T, Mikuni C, Miura A, et al. 2000. Alternating combination chemotherapy C-MOPP (cyclophosphamide, vincristine, procarbazine, prednisone) and ABVd (adriamycin, bleomycin, vinblastine, dacarbazine) in clinical stage II-IV Hodgkin's disease: a multicenter phase II study (JCOG 8905). The Lymphoma Study Group of the Japan Clinical Oncology Group. Jpn J Clin Oncol, 30:146-52.

van den Bent MJ, Delattre J-Y, Brandes AA, et al. 2005. First analysis of EORTC trial 26951, a randomized phase III study of adjuvant PCV chemotherapy in patients with highly anaplastic oligodendroglioma. J Clin Oncol, 23:1503.

van den Bent MJ, Carpentier AF, Brandes AA, et al. 2006. Adjuvant procarbazine, lomustine, and vincristine improves progression-free survival but not overall survival in newly diagnosed anaplastic oligodendrogliomas and oligoastrocytomas: a randomized European Organisation for Research and Treatment of Cancer phase III trial. J Clin Oncol, 24:2715-22.

Yung WK, Albright RE, Olson J, et al. 2000. A phase II study of temozolomide vs. procarbazine in patients with glioblastoma multiforme at first relapse. Br J Cancer, 83:588-93.

Zbinden G. 1980. Unscheduled DNA synthesis in the testis, a secondary test for the evaluation of chemical mutagens. Arch Toxicol, 46:139-49.

Zeller P, Gutmann H, Hegedus B, et al. 1963. Methylhydrazine derivatives, a new class of cytotoxic agents. Experientia, 19:129. 